\title{
Dynamics of Infectious Diseases with Media Coverage and Two Time Delay ${ }^{1}$
}

\author{
Fahad Al Basir*, ** \\ Systems Ecology and Ecological Modeling Laboratory Department of Zoology, \\ Visva-Bharati University Shiksha Bhavana, Shantiniketan 731235, India \\ *e-mail:fahadbasir@gmail.com \\ **e-mail: fahadalbasir@yahoo.com \\ Received January 22, 2018
}

\begin{abstract}
An epidemic model is formulated and analysed on the prevalence of infectious diseases using awareness campaign driven by media with the aim to investigate the effect of awareness and delay on disease outbreak. Two time-delay factors are considered, one is for the time lag in reporting number of infected individuals and another is for the delay between the awareness campaign and the time of taking measures by susceptible individual. The system exhibits two equilibria: the disease-free equilibrium and endemic equilibrium. The disease-free equilibrium is stable for any delay when the basic reproduction number is less than unity. The endemic equilibrium exhibits Hopf-bifurcation for both the delays. Numerical simulations prove the results of analytical outcomes and the significance of awareness and delay in controlling infectious diseases.
\end{abstract}

Keywords: infectious disease, awareness program, basic reproduction number, prior and subsequent delay, hopf-bifurcation, Numerical simulation

DOI: $10.1134 / \mathrm{S} 2070048219010071$

\section{INTRODUCTION}

Infectious diseases could be a warning to society as they cause mortality, disability and socioeconomic disruption for millions of individuals. Ebola, Tuberculosis (TB), Pneumonia, Cholera, Malaria, HIV/AIDS etc. are the major infectious diseases we have noticed. Every year, more than 11 million people die due to infectious disease including premature and young children deaths [1].

Generally, the spreads of an infectious disease is reported by the media, such as television programs, newspapers, radio or online social networks, whenever it outbreaks. The reports and daily updates of the infections and deaths etc. have important impacts on the necessity of control of the epidemic [2-4]. Thus, media has an important role in minimizing and controlling of an infectious disease outbreak. Media coverage helps in changing behavior related to public health. It makes the population up to date with the disease and also suggest the necessary controlling methods [7, 12]. Aware people adopts these practice so that their behavioral chances of becoming infected ones are minimized [5]. Moreover, these changes can make a difference of the size of the epidemic. Awareness campaign focus upon increasing peopleâ ${ }^{\mathrm{TM}} \mathrm{s}$ knowledge about the disease transmission and facilitate measures that can reduce chances of being infected [8, 11]. Thus, by conducting awareness campaigns through media, human behavior can be enhanced towards the disease [10].

Role of awareness programs in the epidemic outbreak have been addressed by many researchers through mathematical modeling [14-16] and the references therein. In many of the studies, SIS type epidemic models are proposed considering the density of the awareness program as a separate variable [18, $19,21]$. A model was formulated in [17], for sexually transmitted infections with media coverage assuming that the whole population is aware of risk whereas a certain proportion chooses to respond by limiting their contacts with the infective population. As a result, the spread of infection and the number of infected individuals reduced. Another model was proposed in [9], where aware susceptible are also vulnerable, as unaware susceptible, to the disease and become infected but with lower rate than that of unaware suscep-

\footnotetext{
${ }^{1}$ The article is published in the original.
} 
tible. This infection rate is very low and has no significant impact on the dynamic. For this, in this article, we have ignore the fact.

Besides, one of the most important problems for epidemiological model is to investigate the effect of time delays on the dynamics of the systems [23]. Time delay is a common phenomenon in population dynamics and need to be focused for practical purposes while formulating mathematical models [24, 25]. Time delay may arise in awareness campaigns also. Misra et al. [22] have addressed a delay induced mathematical model for controlling infectious diseases with awareness programs and oscillations were raised in the system due to the cause of delay. Zhao et al. [26] proposed a SIRS type epidemic model incorporating media campaign with time delay. Samanta [27] has also proposed an epidemic model for infectious disease with awareness program and investigate the effect of delay in reporting infected individual. But he has only considered the reporting delay. Zuo and Liu [28] proposed and analysed an epidemic model with the effect of awareness programs and time delay assuming that the growth rate of cumulative density of awareness programs increases at a rate proportional to the infective population. They have considered a delay as people take some time after the media reports the disease to protect them. Agaba et al. [20] have also proposed a mathematical model with delay due to the time taken by unaware susceptible to become aware. Greenhalgh et al. [21] have introduced two delays, one in reporting of infected cases, and another delay in representing the loss of disease awareness after a fixed period of time and established that both time delays can lead to a destabilisation of the endemic equilibrium. In this article, we have considered two delays: one is for delay in reporting the infected cases to the hospital or health organisation i.e. prior delay, another delay is for the delay unaware susceptible take to become aware after an advertisement is made i.e. subsequent delay. In my knowledge, this is the first work considering both prior and subsequent delay.

The article is organized as follows. In the very next section, the mathematical model is proposed using delay differential equation and some mathematical properties are discussed. Then stability of the disease-free and the endemic equilibrium are analysed in Section 4. Further, some numerical examples are performed to validate the analytical findings in Section 5. In Section 6 we conclude the findings from this study.

\section{THE MATHEMATICAL MODEL}

The following assumptions are taken to formulate the model. Let $S(t)$ and $I(t)$ be the density of the susceptible and infected population respectively at time $t$. The disease is transmitted from infected to susceptible individuals following a mass action functional form. $M(t)$ is the cumulative density of media campaign at any time $t$. The total susceptible population is divided into two subclasses: the unaware susceptible population $S_{u}$ and the aware susceptible population $S_{a}$. As the awareness program is broadcasted, people eventually respond to it and change their behavior to alter their susceptibility. Usually, aware susceptible individuals become infected with lower rate than that of unaware susceptible individuals. It is also assumed that infected individuals recover through treatment. After recovery, a fraction $p$ of recovered people will join the aware susceptible class, whereas the remaining fraction, $1-p(=q)$, will join unaware susceptible class.

The parameters used are described as follows. $\pi$ is the constant recruitment in susceptible population, $\beta$ is the disease transmission rate, $d$ is the natural mortality rate of the population, $e$ is the disease induced mortality rate of infected population and $r$ is the recovery rate.

Here, it is considered that all the newly recruited individuals are unaware. It is assumed that the rate of being aware is proportional to the number of infected individuals reported by the media and/or health organization, whereas the depletion of the aware state is inversely proportional to the number of cases. It is also assumed that unaware susceptible population becomes aware at the rate $\alpha$, where $\alpha$ is the rate at which unaware susceptible individual becomes aware susceptible and is the function of media campaign, $M(t)$. Aware susceptible population becomes unaware at the rate $\lambda$ due to memory fading and/or carelessness. Awareness programs are implemented proportionally with the change of unaware infective individuals at a rate, $\eta$ and cut down at a rate, $\eta_{0}$, due to their ineffectiveness.

There might be some delay in reporting the number of infected individuals in hospital or health center. So, a time delay, $\tau_{1}>0$, occurs in organising the awareness programs. On the other hand, when awareness campaigns are made, people cannot take measures of the media campaigns to protect themselves in time. The need some time to become aware of the disease. So another time delay, $\tau_{2}>0$, occurs that represents 
the time lag that susceptible human take to become aware. On the above discussion, the following system is obtained:

$$
\begin{gathered}
\frac{d S_{u}}{d t}=\pi-\beta S_{u} I-\alpha S_{u} M\left(t-\tau_{2}\right)+\lambda S_{a}-d S_{u}+q r I, \\
\frac{d S_{a}}{d t}=\alpha S_{u} M\left(t-\tau_{2}\right)-\lambda S_{a}-d S_{a}+p r I, \\
\frac{d I}{d t}=\beta S_{u} I-(d+e+r) I, \\
\frac{d M}{d t}=\eta I\left(t-\tau_{1}\right)-\theta M,
\end{gathered}
$$

with the initial conditions: $S_{u}(\phi)=S_{0 u}>0, S_{a}(\phi)=S_{0 a}>0, I(\phi)=I_{0}>0, M(\phi)=M_{0}>0$, where $\phi \in[-\tau, 0], \tau=\min \left(\tau_{1}, \tau_{2}\right)$. For the analysis of model (1), we need the region of attraction, which is given by the set:

$$
B=\left\{\left(S_{u}, S_{a}, I, M\right) \in R_{+}^{4}: 0 \leq S_{u}, S_{a}, I \leq \frac{\pi}{d}, M \leq \frac{\eta \pi}{\theta d}\right\}
$$

\subsection{Non-negativity of solutions}

Biologically, positivity implies the survival of the populations. To proof this, the methods of Bodnar [30] and Yang et al. [31] have been followed, and provide the following theorem:

Theorem 2.1 All the solution of (1) with the positive initial conditions are positive.

Proof. Using the lemma in [30, 31], we show that the solution of the system of Eq. (1) exists in the region $\mathbb{R}_{+}^{4}$ and all solutions remain non-negative for all $t>0$.

It is easy to check in system (2.1) that whenever choosing $X(\lambda) \in R_{+}$such that $S_{u}=0, S_{a}=0, I=0, M=0$, then

$$
\left.f_{i}(X)\right|_{x_{i}=0, X \in R_{+}^{4}} \geq 0,
$$

with $x_{1}(t)=S_{u}(t), x_{2}(t)=S_{a}(t), x_{3}(t)=I(t), x_{4}(t)=M(t)$ and $f_{i}$ are right sides of system (1).

Using the lemma in [31], theorem in [30], we can conclude that any solution of (1) with $X(\lambda) \in C$, say $X(t)=X(t, X(\lambda))$, is such that $X(\lambda) \in R_{+}^{4}$ for all $t \geq 0$. Hence the solution of the system of system (2.1) exist in the region $R_{+}^{4}$ and all solutions remain non-negative for all $t>0$. Therefore, the positive octant $\mathbb{R}_{+}^{4}$ is an invariant region.

\section{STABILITY OF THE SYSTEM WITHOUT DELAY}

In this section, we analyse the equilibria and stability of the non-delayed system. The system, without delay, has two equilibria viz.

i. the disease-free equilibrium, $E^{0}$, which always exists and is in the form:

$$
E^{0}\left(S_{u}^{0}, S_{a}^{0}, I^{0}, M^{0}\right)=\left(\frac{\pi}{d}, 0,0,0,0\right) .
$$

ii. the endemic equilibrium, $E^{*}$, is of the form: $E^{*}\left(S_{u}^{*}, S_{a}^{*}, I^{*}, M^{*}\right)$, in which

$$
\begin{gathered}
S_{u}^{*}=\frac{d+e+r}{\beta}, \quad S_{a}^{*}=\frac{M^{*}[\beta p r \theta+\eta \alpha(d+e+r)]}{\beta \eta}, \\
I^{*}=\frac{\theta M^{*}}{\eta}, \text { and } M^{*}=\frac{\beta \pi-d(d+e+r)}{(d+e+r) \eta \alpha+\beta(d+e)} .
\end{gathered}
$$

It is clear that the endemic equilibria $E^{*}$ exists for

$$
\beta \pi-d(d+e+r)>0 .
$$


The Jacobian matrix at any equilibrium point is given by:

$$
J=\left(\begin{array}{cccc}
J_{1} & 0 & J_{3} & J_{4} \\
J_{5} & J_{6} & J_{7} & J_{8} \\
J_{9} & 0 & J_{10} & 0 \\
0 & 0 & J_{11} & J_{12}
\end{array}\right),
$$

where,

$$
\begin{gathered}
J_{1}=-\beta I-\alpha M-d, \quad J_{3}=q r-\beta S_{u}, \quad J_{4}=-J_{8}=-\alpha S_{u}, \quad J_{5}=\alpha M, \quad J_{6}=-d, \quad J_{7}=p r, \\
J_{9}=\beta I, \quad J_{10}=\beta S_{u}-(d+e+r), \quad J_{11}=\eta, \quad J_{12}=-\theta .
\end{gathered}
$$

\subsection{Stability}

At the disease free equilibria, $E^{0}$, the characteristic equation of the Jacobian matrix has only negative eigenvalues if $\beta \pi-d(d+e+r)<0$. Thus we have the following theorem:

Theorem 3.1. Define $R_{0}=\frac{\beta \pi}{d(d+e+r)}$ as the basic reproduction number for model system. Then the disease free equilibrium $E^{0}$ is stable if $R_{0}<1$ and unstable for $R_{0}>1$. The endemic equilibrium $E^{*}$ exists for $R_{0}>1$.

At $E^{*}$ the characteristic equation is given by:

$$
\rho^{4}+\sigma_{1} \rho^{3}+\sigma_{2} \rho^{2}+\sigma_{3} \rho+\sigma_{4}=0
$$

where,

$$
\begin{gathered}
\sigma_{1}=-\left[J_{1}^{*}+J_{6}^{*}+J_{10}^{*}+J_{12}^{*}\right], \\
\sigma_{2}=J_{1}^{*} J_{6}^{*}-J_{3}^{*} J_{9}^{*}+J_{1}^{*} J_{10}^{*}+J_{6}^{*} J_{10}^{*}+J_{1}^{*} J_{12}^{*}+J_{6}^{*} J_{12}^{*}+J_{10}^{*} J_{12}^{*}, \\
\sigma_{3}=+J_{3}^{*} J_{6}^{*} J_{9}^{*}-J_{1}^{*} J_{6}^{*} J_{10}^{*}-J_{4}^{*} J_{9}^{*} J_{11}^{*} \\
-J_{1}^{*} J_{6}^{*} J_{12}^{*}+J_{3}^{*} J_{9}^{*} J_{12}^{*}-J_{1}^{*} J_{10}^{*} J_{12}^{*}-J_{6}^{*} J_{10}^{*} J_{12}^{*}, \\
\sigma_{4}=J_{4}^{*} J_{6}^{*} J_{9}^{*} J_{11}^{*}-J_{3}^{*} J_{6}^{*} J_{9}^{*} J_{12}^{*}+J_{1}^{*} J_{6}^{*} J_{10}^{*} J_{12}^{*} .
\end{gathered}
$$

where, $J_{1}^{*}=-\beta I^{*}-\alpha M^{*}-d, J_{3}^{*}=q r-\beta S_{u}^{*}, J_{4}^{*}=-J_{8}^{*}=-\alpha S_{u}^{*}, J_{5}^{*}=\alpha M^{*}, J_{6}^{*}=-d, J_{7}^{*}=p r, J_{9}^{*}=\beta I^{*}$, $J_{10}^{*}=\beta S_{u}^{*}-(d+e+r), J_{11}^{*}=\eta, J_{12}^{*}=-\theta$.

According to Routh-Hurwitz criterion, all the eigenvalues of the Jacobian matrix at $E^{*}$ are negative or have negative real part if:

$$
\sigma_{1}>0, \quad \sigma_{4}>0, \quad \sigma_{1} \sigma_{2}-\sigma_{3}>0, \quad\left(\sigma_{1} \sigma_{2}-\sigma_{3}\right) \sigma_{3}-\sigma_{1}^{2} \sigma_{4}>0 .
$$

Proposition 1. The endemic equilibrium point $E^{*}$ is stable if the conditions given in (6) are satisfied.

\section{STABILITY OF THE SYSTEM WITH DELAYS}

In this section, the local stability of the delayed system (1) is studied around the coexisting equilibrium point only. Without loss of generality it is assumed that $E^{*}$ be the interior equilibrium point of the system

(1). The expressions of $S_{u}^{*}, S_{a}^{*}, I^{*}, M^{*}$ are already obtained in the previous section. We are now interested the local asymptomatic stability of the infected steady state $E^{*}$ for the delayed system. Linearizing the system (1) about $E^{*}$, we get

$$
\frac{d X}{d t}=J X(t)+G X\left(t-\tau_{1}\right)+H X\left(t-\tau_{2}\right)
$$


where, $F, G, H$ are $4 \times 4$ matrices, given as below:

$$
\begin{gathered}
J=\left[J_{i j}\right]=\left(\begin{array}{cccc}
-\beta-\alpha M^{*}-d & 0 & -\beta S_{u}^{*}+q r & 0 \\
\alpha M^{*} & -d & p r & 0 \\
\beta I^{*} & 0 & \beta S_{u}^{*}-(d+e+r) & 0 \\
0 & 0 & 0 & -\theta
\end{array}\right), \\
G=\left[G_{i j}\right]=\left(\begin{array}{cccc}
0 & 0 & 0 & -\alpha S_{u}^{*} \\
0 & 0 & 0 & \alpha S_{u}^{*} \\
0 & 0 & 0 & 0 \\
0 & 0 & 0 & 0
\end{array}\right), \\
H=\left[\begin{array}{llll}
0 & 0 & 0 & 0 \\
0 & 0 & 0 & 0 \\
0 & 0 & 0 & 0 \\
0 & 0 & \eta & 0
\end{array}\right) .
\end{gathered}
$$

The characteristic equation of system (1) is given by,

$$
\Delta(\xi)=\left|\xi I-J-e^{-\xi \tau_{1}} G-e^{-\xi \tau_{2}} H\right|=0 .
$$

This equation can be written as,

$$
\psi\left(\xi, \tau_{1}, \tau_{2}\right)=\xi^{4}+a_{1} \xi^{3}+a_{2} \xi^{2}+a_{3} \xi+a_{4}+e^{-\xi\left(\tau_{1}+\tau_{2}\right)}\left[b_{1} \xi+b_{2}\right]=0 .
$$

where

$$
\begin{gathered}
a_{1}=-\left[J_{11}+J_{22}+J_{33}+J_{44}\right], \\
a_{2}=-J_{12} J_{21}+J_{11} J_{22}-J_{13} J_{31}+J_{11} J_{33}+J_{22} J_{33}+J_{11} J_{44}+J_{22} J_{44}+J_{33} J_{44}, \\
a_{3}=J_{13} J_{22} J_{31}-J_{12} J_{23} J_{31}+J_{12} J_{21} J_{33}-J_{11} J_{22} J_{33}+J_{12} J_{21} J_{44} \\
-J_{11} J_{22} J_{44}+J_{13} J_{31} J_{44}-J_{11} J_{33} J_{44}-J_{22} J_{33} J_{44}, \\
a_{4}=-J_{13} J_{22} J_{31} J_{44}+J_{12} J_{23} J_{31} J_{44}-J_{12} J_{21} J_{33} J_{44}+J_{11} J_{22} J_{33} J_{44}, \\
b_{1}=-G_{14} J_{31} J_{43}, \quad b_{2}=\left[G_{14} J_{22} J_{31} H_{43}-J_{12} G_{24} J_{31} H_{43}\right] .
\end{gathered}
$$

The coexisting equilibrium point $E^{*}$ will be locally asymptotically stable if all the roots of the corresponding characteristic Eq. (8) are negative or having negative real parts. The classical Routh-Hurwitz criterion cannot be used to investigate the stability of the system as the Eq. (8) is a transcendental equation in $\xi$. It is known that $E^{*}$ is locally asymptotically stable if all the roots of the corresponding characteristic equation have negative real parts and unstable if purely imaginary roots exist. The following cases may arise.

Case I: When $\tau_{1}=\tau_{2}=0$

In absence of both the delays the characteristics equation (8) becomes,

$$
\xi^{4}+a_{1} \xi^{3}+a_{2} \xi^{2}+\left(a_{3}+b_{1}\right) \xi+\left(a_{4}+b_{2}\right)=0 .
$$

Employing Routh Hurwitz criteria for sign of roots we have the same results as in non delayed system analysis.

Case II: $\tau_{1}>0, \tau_{2}>0$

Taking $\tau_{1}+\tau_{2}=\bar{\tau}$, the characteristic equation becomes,

$$
\psi(\xi, \tau)=\xi^{4}+a_{1} \xi^{3}+a_{2} \xi^{2}+a_{3} \xi+a_{4}+e^{-\xi \tau}\left[b_{1} \xi+b_{2}\right]=0,
$$

A necessary condition for a stability changes of $E^{*}$ is that the characteristic Eq. (11) should have purely imaginary solutions. Let $i \theta$ be a root of Eq. (11) and from which we get,

$$
b_{1} \sin \theta \bar{\tau}+b_{2} \cos \theta \bar{\tau}=-\theta^{4}+a_{2} \theta^{2}-a_{4},
$$




$$
b_{1} \cos \theta \bar{\tau}-b_{2} \cos \theta \bar{\tau}=a_{1} \theta^{3} .
$$

Squaring and adding above two equations,

$$
\theta^{8}+\alpha_{1} \theta^{6}+\alpha_{2} \theta^{4}+\alpha_{3} \theta^{2}+\alpha_{4}=0 .
$$

Simplifying and substituting $\theta^{2}=l$ in above equation, we get the following equation

$$
l^{4}+\alpha_{1} l^{3}+\alpha_{2} l^{2}+\alpha_{3} l+\alpha_{4}=0,
$$

where

$$
\begin{gathered}
\alpha_{1}=a_{1}^{2}-2 a_{2}, \\
\alpha_{2}=a_{2}^{2}+2 a_{4}, \\
\alpha_{3}=-2 a_{2} a_{4}, \\
\alpha_{4}=a_{4}^{2}-\left(b_{1}^{2}+b_{2}^{2}\right) .
\end{gathered}
$$

It may be noted that Eq. (15) will have negative real part if and only if Routh-Hurwitz criterion is satisfied and hence Eq. (14) will have no purely imaginary root. Thus, we have the following proposition.

Proposition 2. In case of delay model system (8), the infected steady state $E^{*}$ will be locally asymptotically stable for all $\bar{\tau}>0$, if the following conditions are satisfied:

$$
\alpha_{i}>0, \quad i=1,2,3,4
$$

If $\alpha_{4}<0$ holds then Eq. (15) will admit at least one positive root. If $\theta_{0}$ be a positive root of (15), then equation $\theta^{8}+\alpha_{1} \theta^{6}+\alpha_{2} \theta^{4}+\alpha_{3} \theta^{2}+\alpha_{4}=0$ will have a purely imaginary root $\pm i \theta_{0}$ corresponding to the delay $\bar{\tau}$. By Butler's lemma, [32] the endemic equilibrium $E^{*}$ remains stable for $\bar{\tau}<\bar{\tau}^{*}$. Now we evaluate the critical value of $\bar{\tau}$ for which the delayed system (8) remain stable.

From Eq. (12),

$$
\bar{\tau}^{*}=\frac{1}{\theta_{0}} \cos ^{-1}\left[\frac{b_{2}\left(-\theta_{0}^{4}+a_{2} \theta_{0}^{2}-a_{4}\right)+b_{1} a_{1} \theta_{0}^{3}}{b_{1}^{2}+b_{2}^{2}}\right]+\frac{2 \pi n}{\theta_{0}}, \quad n=0,1,2,3, \ldots
$$

From the above analysis we have the following theorem.

Theorem 4.1. If $\alpha_{4}<0$ is satisfied then the steady state $E^{*}$ is locally asymptotically stable for $\bar{\tau}<\bar{\tau}^{*}$ and becomes unstable for $\bar{\tau}>\bar{\tau}^{*}$. Furthermore, the system will undergo a Hopf-bifurcation at $E^{*}$ when $\bar{\tau}=\bar{\tau}^{*}$ provided $4 \theta_{0}^{6}+A_{1} \theta_{0}^{4}+A_{2} \theta_{0}^{2}+A_{3}>0$,

were,

$$
\begin{gathered}
A_{1}=3 a_{1}-6 a_{2}, \\
A_{2}=2 a_{2}+4 a_{4}-4 a_{1} a_{3}, \\
A_{3}=a_{3}^{2}-2 a_{2} a_{4}-b_{1}^{2} .
\end{gathered}
$$

Proof. Differentiating (11) with respect to $\bar{\tau}$ we get:

$$
\frac{d \bar{\tau}}{d \xi}=\frac{4 \xi^{3}+3 a_{1} \xi^{2}+2 a_{2} \xi+a_{3}}{b_{1} \xi^{2}+b_{2}} e^{\xi \bar{\tau}}+\frac{b_{1}}{b_{1} \xi^{2}+b_{2} \xi}-\frac{\bar{\tau}}{\xi} .
$$

Now, using the relation (12) one can obtain:

$$
\begin{gathered}
\operatorname{sgn}\left[\frac{d(\operatorname{Re} \xi}{d \bar{\tau}}\right]_{\bar{\tau}=\bar{\tau}^{*}}=\operatorname{sgn}\left[\operatorname{Re}\left(\frac{d \xi}{d \bar{\tau}}\right)^{-1}\right]_{\xi=i \theta_{0}}, \\
=\operatorname{sgn}\left[\frac{4 \theta_{0}^{6}+A_{1} \theta_{0}^{4}+A_{2} \theta_{0}^{2}+A_{3}}{b_{1} \theta_{0}^{2}+b_{2}^{2}}\right] .
\end{gathered}
$$


Now sgn $\left[\frac{d(\operatorname{Re} \xi}{d \bar{\tau}}\right]_{\bar{\tau}=\bar{\tau}^{*}}>0$ if $4 \theta_{0}^{6}+A_{1} \theta_{0}^{4}+A_{2} \theta_{0}^{2}+A_{3}>0$ i.e. the transversality condition holds and the system undergoes Hopf bifurcation at $\bar{\tau}=\bar{\tau}^{*}$.

Case III: when $\tau_{1}>0, \tau_{2}=0$

In this case we consider no delay in vector infection i.e $\tau_{2}=0$, then the characteristic equation becomes,

$$
\psi\left(\xi, \tau_{1}\right)=\xi^{4}+a_{1} \xi^{3}+a_{2} \xi^{2}+a_{3} \xi+a_{4}+e^{-\xi \tau_{1}}\left[b_{1} \xi+b_{2}\right]=0 .
$$

Let $i \omega$ be a root of equation (16) and from which we get,

$$
\begin{gathered}
b_{1} \sin \omega \tau_{1}+b_{2} \cos \omega \tau_{1}=-\omega^{4}+a_{2} \omega^{2}-a_{4}, \\
b_{1} \cos \omega \tau_{1}-b_{2} \cos \omega \tau_{1}=a_{1} \omega^{3} .
\end{gathered}
$$

Squaring and adding above two equations,

$$
\omega^{8}+\alpha_{1} \omega^{6}+\alpha_{2} \omega^{4}+\alpha_{3} \omega^{2}+\alpha_{4}=0 .
$$

Simplifying and substituting $\omega^{2}=l$ in Eq. (18) we get the following equation

$$
l^{4}+A_{1} l^{3}+A_{2} l^{2}+A_{3} l+A_{4}=0,
$$

where

$$
\begin{gathered}
A_{1}=a_{1}^{2}-2 a_{2}, \\
A_{2}=a_{2}^{2}+2 a_{4}, \\
A_{3}=-2 a_{2} a_{4}, \\
A_{4}=a_{4}^{2}-\left(b_{1}^{2}+b_{2}^{2}\right) .
\end{gathered}
$$

It may be noted that Eq. (22) will have negative real part if and only if Routh-Hurwitz criterion is satisfied and hence Eq. (17) will have no purely imaginary root. Thus, we have the following proposition.

Proposition 3. In case of delay model system (8), the infected steady state $E^{*}$ will be locally asymptotically stable for all $\tau_{1}>0$, if the following conditions are satisfied:

$$
A_{i}>0, \quad i=1,2,3,4
$$

Let, $\pm i \omega_{0}$ imaginary root corresponding to the delay $\tau_{1}$. Then, we have the following theorem.

Theorem 4.2. If $\alpha_{4}<0$ is satisfied then the steady state $E^{*}$ is locally asymptotically stable for $\tau_{1}<\tau_{1}^{*}$ and becomes unstable for $\tau_{1}>\tau_{1}^{*}$. Furthermore, the system will undergo a Hopf-bifurcation at $E^{*}$ when $\tau_{1}=\tau_{1}^{*}$ provided $4 \omega_{0}^{6}+A_{1} \omega_{0}^{4}+A_{2} \omega_{0}^{2}+A_{3}>0$,

where,

$$
\begin{gathered}
A_{1}=3 a_{1}-6 a_{2}, \\
A_{2}=2 a_{2}+4 a_{4}-4 a_{1} a_{3}, \\
A_{3}=a_{3}^{2}-2 a_{2} a_{4}-b_{1}^{2} .
\end{gathered}
$$

with

$$
\tau_{1}^{*}=\frac{1}{\omega_{0}} \cos ^{-1}\left[\frac{b_{2}\left(-\omega_{0}^{4}+a_{2} \omega_{0}^{2}-a_{4}\right)+b_{1} a_{1} \omega_{0}^{3}}{b_{1}^{2}+b_{2}^{2}}\right]+\frac{2 \pi n}{\omega_{0}}, \quad n=0,1,2,3, \ldots
$$

Proof. Transversality condition for Hopf-bifurcation: Differentiating (10) with respect to $\tau_{1}$ we get:

$$
\frac{d \tau_{1}}{d \xi}=\frac{4 \xi^{3}+3 a_{1} \xi^{2}+2 a_{2} \xi+a_{3}}{b_{1} \xi^{2}+b_{2}} e^{\xi \tau_{1}}+\frac{b_{1}}{b_{1} \xi^{2}+b_{2} \xi}-\frac{\tau_{1}}{\xi}
$$

Now, using the relation (19) one can obtain: 
(a)
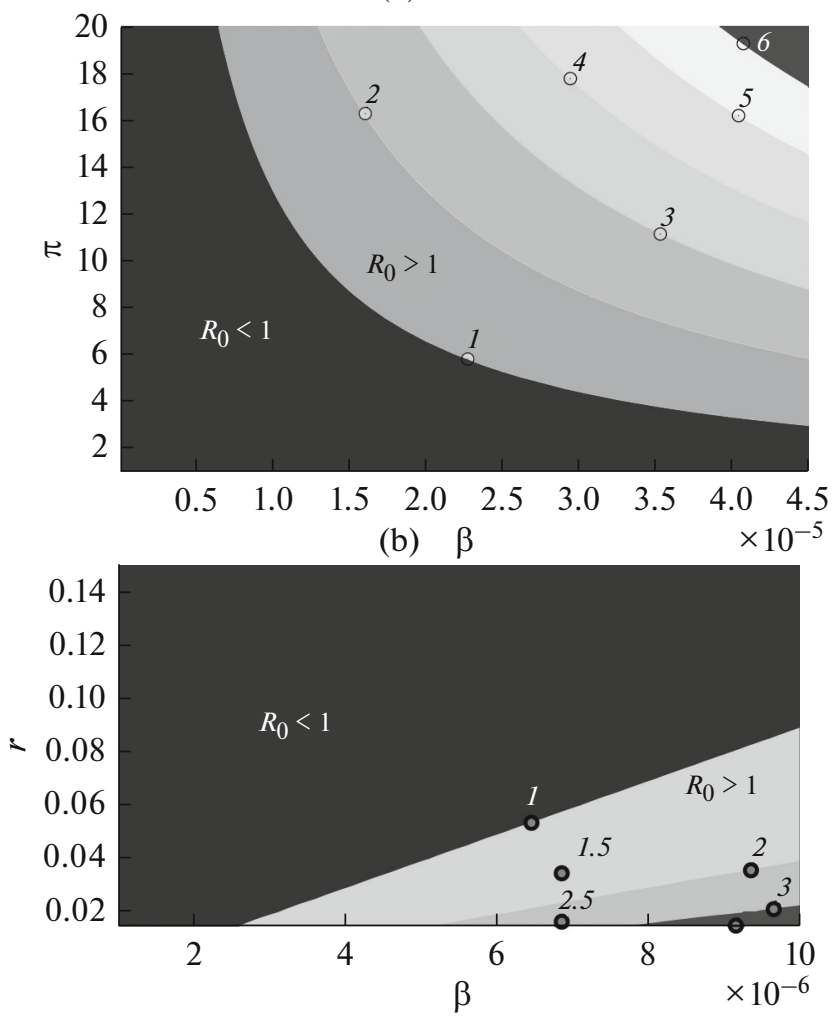

Fig. 1. Contour plot of $R_{0}$ : (a) in $\beta-\pi$ and (b) in $\beta-r$ parameter planes using the parameter values as given in Table 1 .

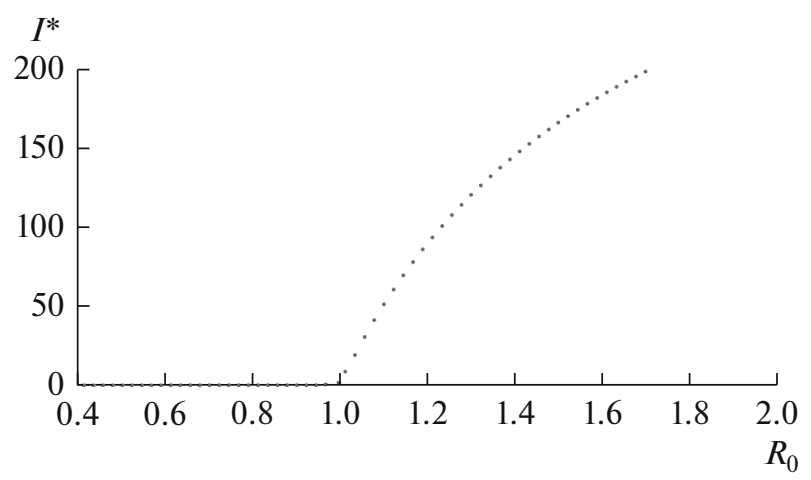

Fig. 2. Transcritical bifurcation: steady state value of infected human, $I$, is plotted against $R_{0}$ taking parameters from Table 1 except $\beta$.

$$
\begin{gathered}
\operatorname{sgn}\left[\frac{d(\operatorname{Re} \xi}{d \tau_{1}}\right]_{\tau_{1}=\tau_{1}^{*}}=\operatorname{sgn}\left[\operatorname{Re}\left(\frac{d \xi}{d \tau_{1}}\right)^{-1}\right]_{\xi=i \omega_{0}}, \\
=\operatorname{sgn}\left[\frac{4 \omega_{0}^{6}+A_{1} \omega_{0}^{4}+A_{2} \omega_{0}^{2}+A_{3}}{b_{1} \omega_{0}^{2}+b_{2}^{2}}\right] .
\end{gathered}
$$



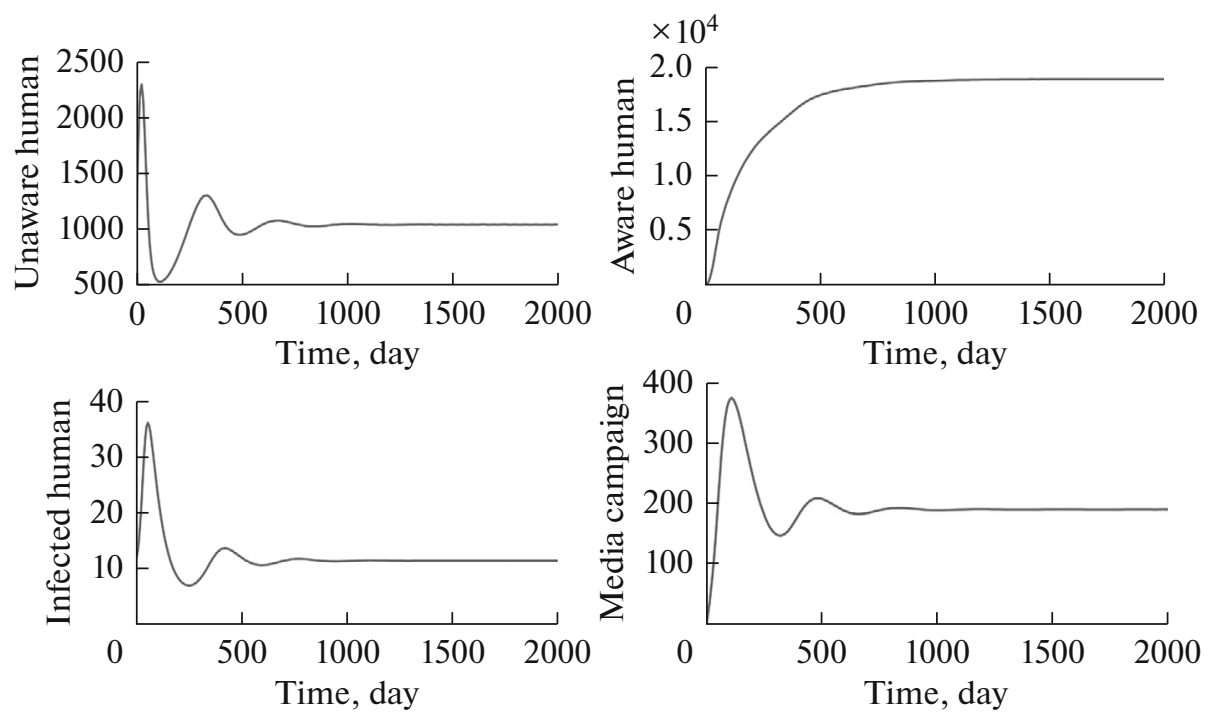

Fig. 3. Time series solution of the system without delay is plotted with the parameter values as given in Table 1 .

Now sgn $\left[\frac{d(\operatorname{Re} \xi}{d \tau_{1}}\right]_{\tau_{1}=\tau_{1}^{*}}>0$ if $4 \omega_{0}^{6}+A_{1} \omega_{0}^{4}+A_{2} \omega_{0}^{2}+A_{3}>0$ i.e. the transversality condition holds and the system undergoes Hopf bifurcation at $\tau_{1}=\tau_{1}^{*}$.

Case IV: When $\tau_{2}>0, \tau_{1}=0$

This analysis is similar to Case III.

\section{NUMERICAL SIMULATION}

In this section, numerical calculations are performed to investigate the dynamics of the system with and without delay to confirm our analytical findings. To study the global dynamic behavior, extensive numerical simulations have been carried out for no time delay firstly then for various values of $\tau_{i}, i=1,2$.

Figure 1 contains the contour plot of $R_{0}$ in two parameter planes. The disease free equilibria is stable in the blue region where $R_{0}$ is lesser one. For the system without delay, the system is endemic and is asymptotically stable (according to Routh-Hurwitz criterion) for $R_{0}>1$.

In Fig. 2, the forward transcritical bifurcation is plotted. Infected human population grows when $\mathrm{R}_{0}$ crosses unity. That means infection occurs when $R_{0}$ is greater than one.

Table 1. List of parameters used for numerical simulations [22, 33-35]

\begin{tabular}{c|l|c}
\hline Parameter & \multicolumn{1}{|c|}{ Definition } & ${\text { Value, } \text { day }^{-1}}^{\mid}$ \\
\hline$\pi$ & Constant recruitment rate & 100 \\
$\beta$ & Disease transmission rate & 0.000025 \\
$\alpha$ & Contact rate between unaware susceptible with media & 0.0005 \\
$\lambda$ & Transfer rate of people from aware individuals to unaware & 0.00025 \\
$d$ & Susceptible class Natural death rate & 0.001 \\
$e$ & Additional death rate due to infection & 0.002 \\
$\eta$ & Rate of implementation of awareness programs & 0.25 \\
$r$ & Rate of recovery through treatment & 0.012 \\
$\theta$ & Fading of interest on awareness program & 0.015 \\
\hline
\end{tabular}




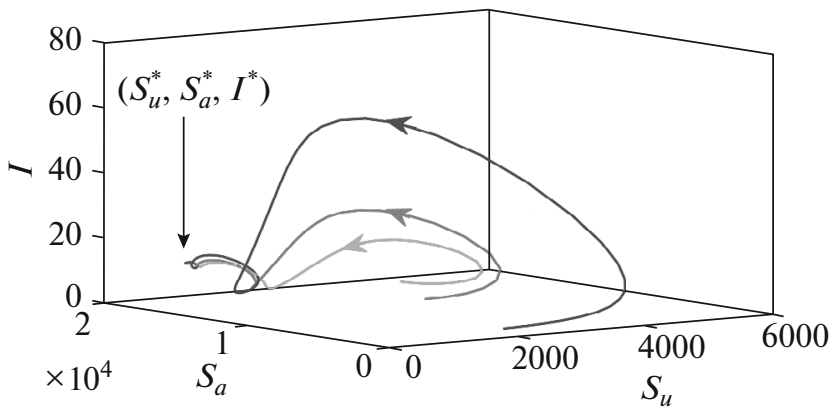

Fig. 4. Time series solution of the system without delay is plotted with the parameter values as given in Table 1 and with three different initial points.
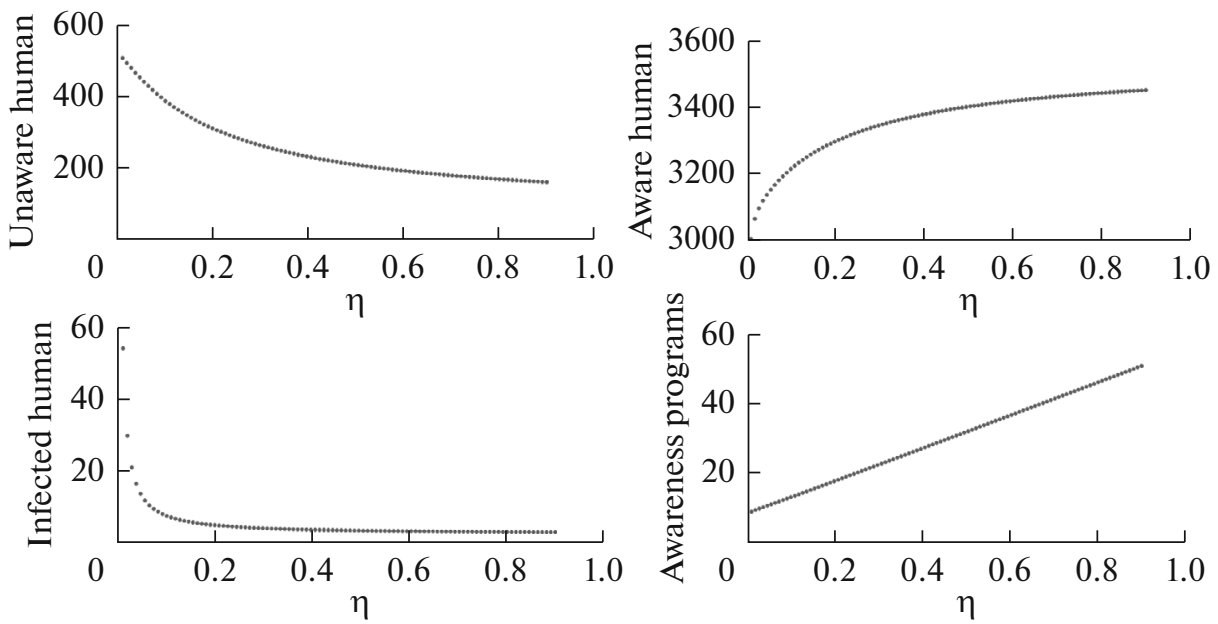

Fig. 5. Steady state value of the system populations are plotted (when the system is stable) as function of $\eta$ (the aware population growth rate). The parameters values are given in Table 1.
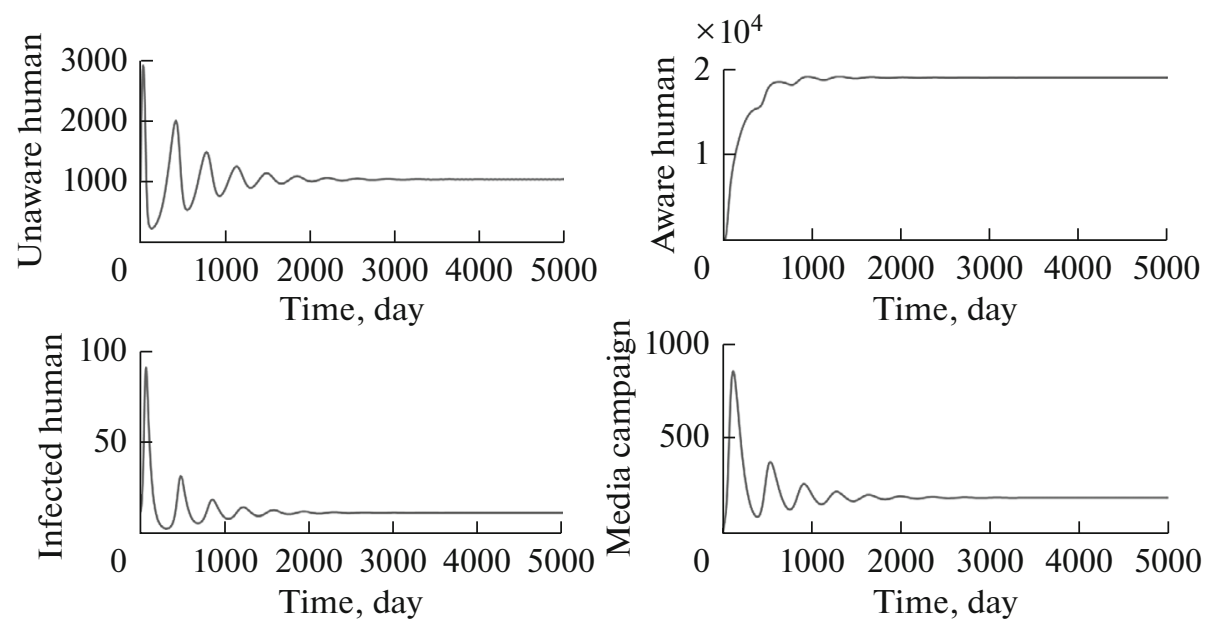

Fig. 6. Time series solution of the system for $\tau_{1}=10, \tau_{2}=10$ is plotted with the set of parameters as in Fig. 1. Here, $\tau_{1}+\tau_{2}=\bar{\tau}<\bar{\tau}^{*}$ and system is stable. 

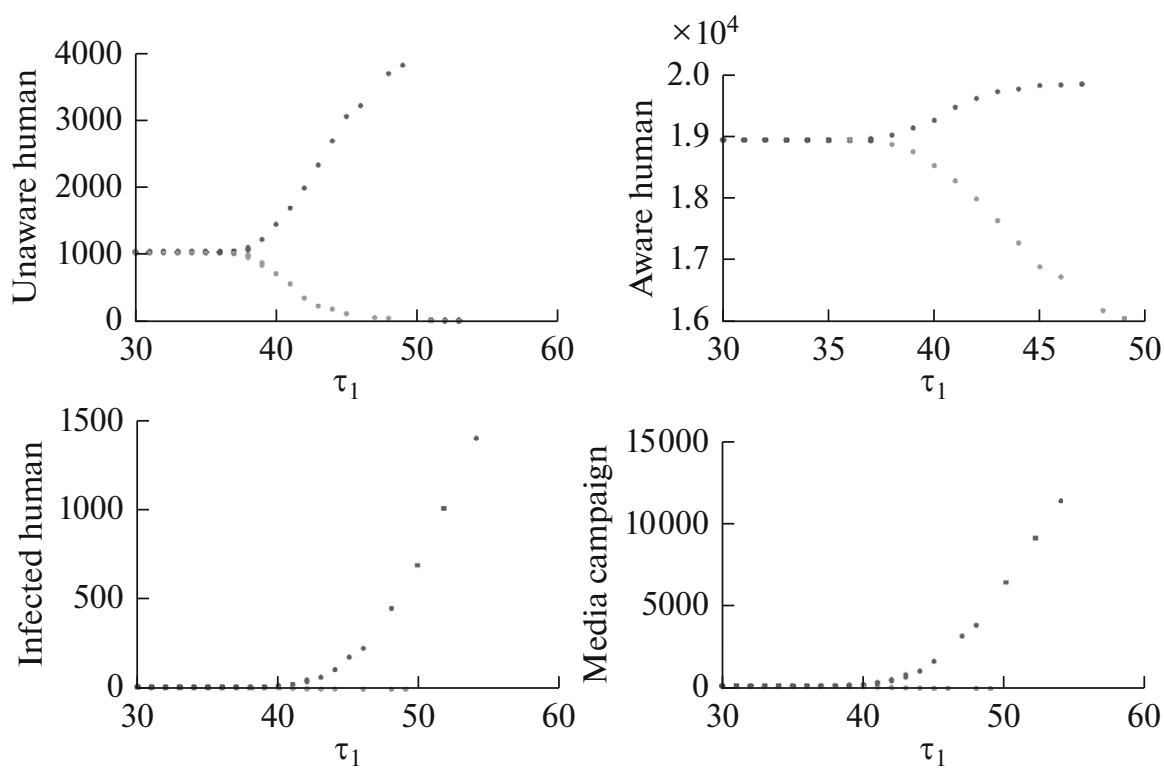

Fig. 7. Bifurcation diagram of the system is plotted considering $\tau_{1}$ as the bifurcating parameter and with $\tau_{2}=0$.
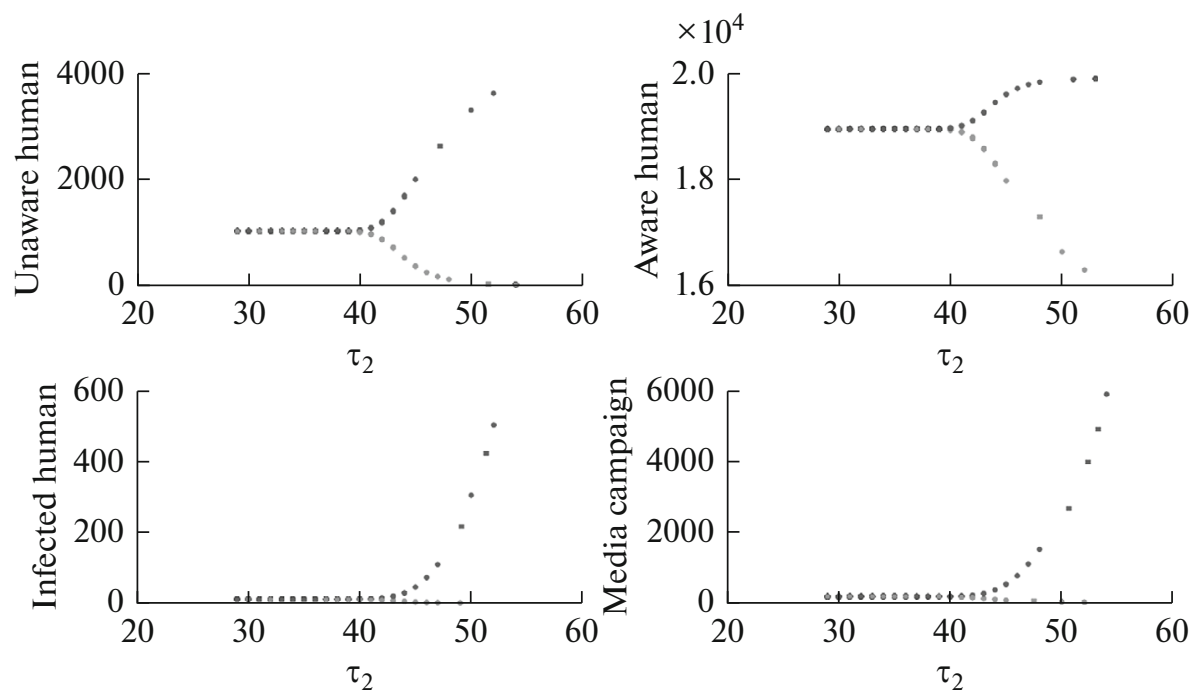

Fig. 8. Bifurcation diagram of the system is plotted. Here, $\tau_{2}$ is the bifurcating parameter and with $\tau_{1}=0$.

In Figure 3, model populations are plotted with time. The system is endemic for the values of the parameters as given in Table 1. The endemic equilibrium is stable according to Proposition 1.

The numerical results presented in Fig. 4 suggest that the equilibrium number of infective individuals decreases with an increase in the rate of being aware. It is also observed that awareness among susceptible population can reduce the epidemic size. Therefore, awareness program can eradicate or control the epidemic. The numerical results presented in Fig. 5 suggest that the equilibrium number of infective individuals decreases with an increase in the rate of being aware. It is also observed that awareness among susceptible population can reduce the epidemic size. Therefore, awareness program can eradicate or control the epidemic.

Considering delay $\left(\tau_{1}=10, \tau_{2}=10\right)$, the system initially oscillates. This indicates that sometimes the number of infective will be high and sometimes low and it may be difficult to make the prediction regarding the size of epidemic. The system takes more time to become stable (see Fig. 6). 

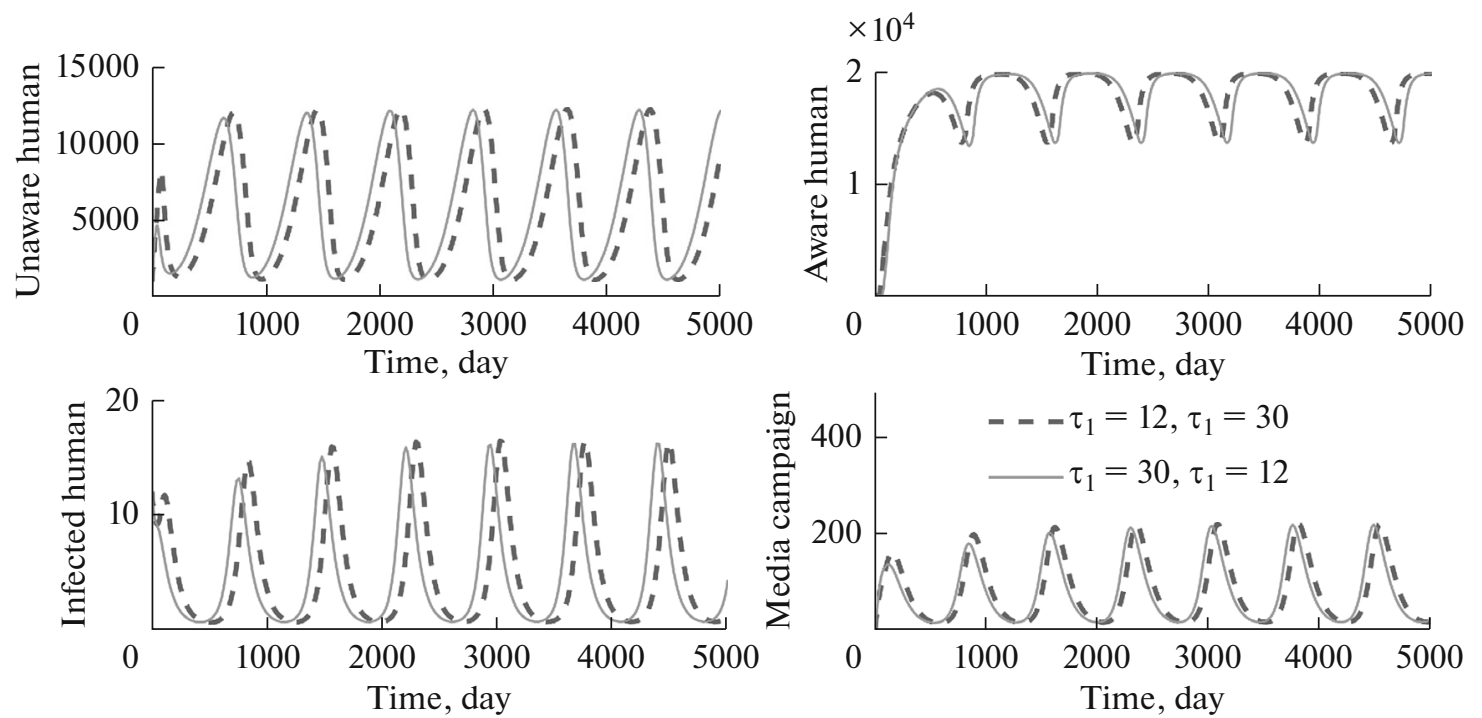

Fig. 9. Time series solution of the system for $\tau_{1}+\tau_{2}>41$ is plotted and periodic solution is observed. Parameter values are given in Table 1.

The variation of system populations for different value of $\tau_{1}$ and $\tau_{2}$ are presented in Figs. 7-9. When the value of delay $\tau_{1}$ exceeds its critical value $\tau_{1}^{*}$, all system variables bifurcate into periodic solutions (see Fig. 7) for $\tau_{2}=0$. Again, as value of delay $\tau_{2}$ exceeds its critical value $\tau_{2}^{*}$, system variables bifurcate into periodic solution (see Fig. 8) for $\tau_{1}=0$. Hence, these figures indicate that equilibrium $E^{*}$ of model system (1) is stable for $\tau_{1}<\tau_{1}^{*}$ and $\tau_{2}<\tau_{2}^{*}$ and unstable if the inequality is reversed. In Fig. 9, for the pair $\tau_{1}=12, \tau_{2}=30$ and $\tau_{1}=30, \tau_{2}=12$, the bifurcating periodic solutions are observed. More clearly, if the delay $\tau_{2}$ is small but the delay $\tau_{2}$ is high and viceversa, periodic oscillation occurs which makes the control process complicated.

\section{DISCISSION AND CONCLUSION}

In this paper, the effect of awareness programs on infectious disease dynamics is analysed considering two time delays. The first time delay, $\tau_{1}$, is due to delay in reporting of infected individual i.e. delay in organizing awareness programs and the second time delay, $\tau_{2}$, is due to the time taken by unaware human to become aware. The existence and stability criteria of the disease free and endemic equilibria have been derived in terms of the basic reproduction number, $R_{0}$. When $R_{0}$ is less than unity, disease cannot persist in the system, whereas for $R_{0}$ above unity, disease coexists in the system and thus, a transcritical bifurcation occurs when $R_{0}$ equals unity. Sufficient conditions for the stability of the endemic equilibrium and the existence of Hopf-bifurcations are obtained for delayed system provided analytically and numerically. It is observed that if the number of campaigns due to the awareness program is increased then the disease transmission amongst the susceptible population declines. However, both delay increases, then the system shows limit cycle oscillations, which pose a challenge to control the epidemic. Interesting thing is that, if the delay in measuring the media campaign is small but the delay in organizing awareness campaign is high and viceversa, periodic oscillation occurs. The government and other health organizations should immediately make people aware about the disease and relevant precautions through the media [1,6]. For smooth controlling of infectious diseases, the should not be much delay in organising awareness campaign and in becoming aware of the disease. The findings from this study will enrich the understanding towards the epidemic outbreak which ultimately useful in controlling the epidemic.

\section{ACKNOWLEDGMENTS}

Fahad Al Basir acknowledges the University Grants Commission, Government of India for Dr DS Kothari Post-Doctoral Fellowship , File no: MA/16-17/0029. 


\section{REFERENCES}

1. J. T. Lau, X. Yang, H. Y. Tusi, and J. H. Kim, "Impacts of SARS on health-seeking behaviors in general population in Hong Kong," Prevent. Med. 41, 454-462, (2005).

2. Liu Maoxing, Yuting Chang, and Lixia Zuo, "Modelling the impact of media in controlling the diseases with a piecewise transmission rate," Discrete Dyn. Nat. Soc. 2016 (2016).

3. J. M. Tchuenche, N. Dube, C. P. Bhunu, R. J. Smith, and C. T. Bauch, "The impact of media coverage on the transmission dynamics of human influenza," BMC Public Health 11 (1), S5, (2011).

4. M. S. Majumder, S. Kluberg, M. Santillana, S. Mekaru, and J. S. Brownstein, "2014 Ebola outbreak: media events track changes in observed reproductive number," PLoS Curr. 7 (2015).

5. S. Collinson and J. M. Heffernan, "Modelling the effects of media during an influenza epidemic," Collinson Heffernan BMC Public Health 14, Article 376 (2014).

6. Jingan Cui, Yonghong Sun, and Huaiping Zhu, "The impact of media on the control of infectious diseases," J. Dyn. Differ. Equations 20 (1), 31-53 (2008).

7. J. H. Jones and M. Salathe, "Early assessment of anxiety and behavioral response to novel swine-origin influenza A (H1N1)," PLoS ONE 4, e8032 (2009).

8. G. J. Rubin, R. Amlôt, L. Page, and S. Wessely, "Public perceptions, anxiety, and behaviour change in relation to the swine flu outbreak: cross sectional telephone survey," Br. Med. J. 339 (2009). doi 10.1136/bmj.b2651

9. S. Samanta, S. Rana, A. Sharma, A.K. Misra, and J. Chattopadhyay, "Effect of awareness programs by media on the epidemic outbreaks: A mathematical model," Appl. Math. Comput. 219, 6965-6977 (2013).

10. J. Cui, Y. Sun, and H. Zhu, "The impact of media on the control of infectious diseases," J. Dyn. Differ. Equations 20, 31-53 (2007).

11. M. Frolich and R. Vazquez Alvarez, "HIV/AIDS knowledge and behaviour: Have information campaigns reduced HIV infection? The case of Kenya," Afr. Dev. Rev. 21 (1), 86-146 (2009).

12. Yongfeng Li and Jingan Cui, "The effect of constant and pulse vaccination on SIS epidemic models incorporating media coverage," Commun. Nonlinear Sci. Numer. Simul. 14 (5), 2353-2365 (2009).

13. C. Sun, W. Yang, J. Arino, and K. Khan, "Effect of media-induced social distancing on disease transmission in a two patch setting," Math. Biosci. 230, 87-95 (2011).

14. A. K. Misra, A. Sharma, and V. Singh, "Effect of awareness programs in controlling the prevalence of an epidemic with time delay," J. Biol. Syst. 19, 389-402 (2011).

15. A. Sharma and A. Misra, "Modeling the impact of awareness created by media campaigns on vaccination coverage in a variable population," J. Biol. Syst. 22, 249-270 (2014).

16. S. Samanta and J. Chattopadhyay, "Effect of awareness program in disease outbreak - a slow-fast dynamics," Appl. Math. Comput. 237, 98-109 (2014).

17. I. Z. Kiss, J. Cassell, M. Recker, and P. L. Simon, "The impact of information transmission on epidemic outbreaks," Math. Biosci. 225, 1-10 (2010).

18. G. O. Agaba, Y. N. Kyrychko, and K. B. Blyuss, "Mathematical model for the impact of awareness on the dynamics of infectious diseases," Math. Biosci. 286, 22-30 (2017).

19. F. A. Basir, "Long term dynamics of infectious diseases with awareness campaign and time delay," WSEAS Trans. Math. 16, 266-275 (2017).

20. G. O. Agaba, Y. N. Kyrychko, and K. B. Blyuss, "Time-delayed SIS epidemic model with population awareness," Ecol. Complexity 31, 50-56 (2017).

21. D. Greenhalgh, S. Rana, S. Samanta, T. Sardar, S. Bhattacharya, and J. Chattopadhyay, "Awareness programs control infectious disease-multiple delay induced mathematical model," Appl. Math. Comput. 251, 539 -563 (2015).

22. F. Al Basir, S. Ray, and E. Venturino, "Role of media coverage and delay in controlling infectious diseases: A mathematical model,” Appl. Math. Comput. 337, 372-385 (2018).

23. M. Lakshmanan and D. V. Senthilkumar, Dynamics of Nonlinear Time-Delay Systems (Springer Science \& Business Media, 2011).

24. F. Al Basir, E. Venturino, S. Ray, and P. K. Roy, "Impact of farming awareness and delay on the dynamics of mosaic disease in Jatropha curcas plantations," Comput. Appl. Math. (2018). doi 0.1007/s40314-018-0675-6

25. F. Al Basir, K. B. Blyuss, and S. Ray, "Modelling the effects of awareness-based interventions to control the mosaic disease of Jatropha curcas," Ecol. Complexity 36, 92-100 (2018).

26. H. Zhao, Y. Lin, and Y. Dai, "An SIRS epidemic model incorporating media coverage with time delay," Comput. Math. Methods Med., Article ID 680743 (2014).

27. S. Samanta, "Effects of awareness program and delay in the epidemic outbreak," Math. Methods Appl. Sci. 40 (5), 1679-1695 (2017).

28. L. Zuo and M. Liu, "Effect of awareness programs on the epidemic outbreaks with time delay," Abstr. Appl. Anal. 2014 (2014).

29. J. Hale, Theory of Functional Differential Equations (Springer-Verlag, New York -Heidelberg -Berlin, 1977). 
30. M. Bodnar, "The nonnegativity of solutions of delay differential equations," Appl. Math. Lett. 13 (6), 91-95 (2000).

31. X. Yang, L. Chen, and J. Chen, "Permanence and positive periodic solution for the single species nonautonomus delay diffusive model," Comput. Math. Appl. 32, 109-116 (1996).

32. H. L. Freedman and V. Sree Hari Rao, "The trade-off between mutual interference and time lags in predatorprey systems," Bull. Math. Biol. 45, 991-1004 (1983).

33. S. Samanta and J. Chattopadhaya, "Effect of awareness program in disease outbreak-A slow-fast dynamics," Appl. Math. Comput. 237, 98-109 (2014).

34. Priti Kumar Roy, Shubhankar Saha, and Fahad Al Basir, "Effect of awareness programs in controlling the disease HIV/AIDS: an optimal control theoretic approach,” Adv. Differ. Equations 2015 (2015).

35. S. D. Hove-Musekwaa and F. Nyabadza, "The dynamics of an HIV/AIDS model with screened disease carriers,” Comput. Math. Methods Med. 10 (4), 287-305 (2009). 